\title{
ORIENTATIONS AND GEOMETRISATIONS OF COMPACT COMPLEX SURFACES
}

\author{
D. KOTSCHICK
}

Every complex manifold carries a canonical orientation, and it is natural to wonder when the underlying topological or smooth manifold carries a complex structure compatible with the other orientation. In [2], Beauville raised this question for compact complex surfaces. He noted that there are a lot of examples, like products of curves, or Hopf surfaces, where the underlying manifold admits an orientation-reversing selfdiffeomorphism. This implies that the signature is zero. Beauville asked if there are any examples of non-zero signature.

In [6], we proved that there are infinitely many pairs of simply connected algebraic surfaces of general type with non-zero signature which are orientation-reversing homeomorphic, but not diffeomorphic. The results of [6] suggested that the only compact complex surfaces with finite fundamental groups for which the underlying smooth (rather than topological) manifolds admit complex structures compatible with the other orientation should be the rational ruled surfaces diffeomorphic to two-sphere bundles over the two-sphere. The main aim of this note is to prove this conjecture. Even without the assumption on the fundamental group, we shall see that all examples must have zero signature. We shall also obtain a complete classification of surfaces of non-negative Kodaira dimension which admit a complex structure compatible with the other orientation. It will turn out that all these surfaces have orientation-reversing selfdiffeomorphisms, and can be characterised in terms of Thurston geometries $[\mathbf{1 0}, \mathbf{5}]$. As a byproduct, we also obtain a classification of all surfaces which admit a Kähler-Einstein metric for each orientation.

All these results are proved by combining an argument of Leung [9] with our arguments from [6], transplanted from Donaldson theory to Seiberg-Witten theory. We refer to $[3, \mathbf{1 1}]$ for the definitions and basic properties of Seiberg-Witten invariants.

We begin with a preliminary result, which is of independent interest.

Proposition 1. Let $X$ be a smooth closed oriented 4-manifold with $b_{2}^{+}(X)>1$ which contains a smoothly embedded 2-sphere $S$ of non-negative selfintersection with $S \neq 0 \in H_{2}(X, \mathbb{Q})$. Then all Seiberg-Witten invariants of $X$ vanish.

Proof. There is a proof of this result in [4]. We give here a simpler proof, modelled on the proof given in [7] for the corresponding result for Donaldson invariants.

Fix a $\operatorname{Spin}^{c}$ structure on $X$. It is easy to see that there is one on $X \# \overline{\mathbb{C} P^{2}}$ which has the same Seiberg-Witten invariant, up to sign (compare Theorem 1.4 in [4], or Proposition 2 in [8]). If $L$ is the auxiliary line bundle for the $\operatorname{Spin}^{c}$ structure of $X$,

Received 15 November 1995.

1991 Mathematics Subject Classification 14J99, 53C55, 53C15.

Bull. London Math. Soc. 29 (1997) 145-149 
the line bundle for the structure on $X \# \overline{\mathbb{C} P^{2}}$ is $L+E$, where $E$ is the exceptional curve.

Using this blowup formula, we reduce to the case when the selfintersection number of $S$ is zero. Note that when a sphere of zero selfintersection is obtained by blowing up points on one of positive selfintersection, then it is automatically non-trivial in $H_{2}(X, \mathbb{Q})$. We blow up once more. In $X \# \overline{\mathbb{C} P^{2}}$, the class $k S+E$ can be represented by a smoothly embedded two-sphere obtained by tubing together $k$ parallel copies of $S$ and tubing the result to $E$. As $k S+E$ has selfintersection -1 , the reflection in its orthogonal complement is realised by a selfdiffeomorphism of $X \# \overline{\mathbb{C} P^{2}}$. The image of $L+E$ under this diffeomorphism is $L+2 k(k S \cdot L-1) S+$ $(2 k S \cdot L-1) E$. Varying $k$, we obtain infinitely many $\operatorname{Spin}^{c}$ structures on $X \# \overline{\mathbb{C} P^{2}}$ which have the same Seiberg-Witten invariant (up to sign) as the $\operatorname{Spin}^{c}$ structure of $X$ that we started with. However, on every manifold there are at most finitely many $\operatorname{Spin}^{c}$ structures with non-zero invariants $[11,3]$. Thus all invariants of $X$ must vanish.

Remark 1. In the case when $b_{2}^{+}(X)=1$, the conclusion of Proposition 1 holds, with the same proof, as long as at the end, after making all the necessary blowups, one is dealing with a manifold with $b_{2}^{-}(X)+4 b_{1}(X) \leqslant 9$. This assumption ensures that the Seiberg-Witten invariants are independent of chambers.

Using Proposition 1, we now generalise Theorem 1 of Leung [9]. We denote by $\bar{X}$ the smooth manifold underlying $X$, but endowed with the orientation opposite to that of $X$.

Theorem 1. Let $X$ be a surface of general type. If $\bar{X}$ admits a non-zero SeibergWitten invariant (of any degree), then $X$ has ample canonical bundle. Further, $c_{1}^{2}(X)$ is even and $X$ has non-negative signature, and $X$ has zero signature if and only if it is uniformised by the polydisk.

Proof. The fact that $X$ has a complex structure implies that $b_{2}^{+}(X)-b_{1}(X)$ is odd. On the other hand, if $\bar{X}$ has a non-zero Seiberg-Witten invariant, $b_{2}^{-}(X)-b_{1}(X)$ is odd because the dimension of the Seiberg-Witten moduli spaces on $\bar{X}$ must be even. Thus $c_{1}^{2}(X)=4+5\left(b_{2}^{+}(X)-b_{1}(X)\right)-\left(b_{2}^{-}(X)-b_{1}(X)\right)$ is even.

Using the Miyaoka-Yau inequality $c_{1}^{2}(X) \leqslant 3 c_{2}(X)$, we see that $b_{2}^{-}(X)>1$, unless $b_{2}^{+}(X)=b_{2}^{-}(X)=1$ and $b_{1}(X)=0$. In both cases, we can apply Proposition 1 to $\bar{X}$ to conclude that $X$ contains no embedded spheres of selfintersection -1 or -2 . (Compare Remark 1 for the case $b_{2}^{+}(X)=b_{2}^{-}(X)=1$.) In particular, $X$ contains no holomorphic spheres with selfintersection -1 or -2 . Thus its canonical bundle is ample.

Let $L$ be the auxiliary line bundle for a $\operatorname{Spin}^{c}$ structure on $\bar{X}$ with respect to which $\bar{X}$ has a non-zero Seiberg-Witten invariant. The expected dimension for the Seiberg-Witten moduli space for $L$ must be non-negative, which means

$$
-\left\langle c_{1}^{2}(L),[X]\right\rangle=\left\langle c_{1}^{2}(L),[\bar{X}]\right\rangle \geqslant 2 \chi(\bar{X})+3 \sigma(\bar{X})=2 \chi(X)-3 \sigma(X) .
$$

By the results of Aubin and Yau on the Calabi conjecture, $X$ has a Kähler-Einstein metric $g$. For such metrics, one has

$$
\frac{1}{32 \pi^{2}} \int_{X} s_{g}^{2} d \operatorname{vol}_{g}=\left\langle c_{1}^{2}(X),[X]\right\rangle=2 \chi(X)+3 \sigma(X),
$$


where $s_{g}$ denotes the scalar curvature function of $g$. We calculate for a solution $(A, \phi)$ of the Seiberg-Witten equations for $L$ on $\bar{X}$ with the metric $g$ :

$$
\begin{aligned}
-\left\langle c_{1}^{2}(L),[X]\right\rangle & =\frac{1}{4 \pi^{2}} \int_{X}\left(\left|F_{A}^{-}\right|^{2}-\left|F_{A}^{+}\right|^{2}\right) d \operatorname{vol}_{g} \leqslant \frac{1}{4 \pi^{2}} \int_{X}\left|F_{A}^{-}\right|^{2} d \operatorname{vol}_{g} \\
& =\frac{1}{32 \pi^{2}} \int_{X}|\phi|^{4} d \operatorname{vol}_{g} \leqslant \frac{1}{32 \pi^{2}} \int_{X} s_{g}^{2} d \operatorname{vol}_{g}=2 \chi(X)+3 \sigma(X) .
\end{aligned}
$$

Here we have used the Seiberg-Witten curvature equation to conclude $\left|F_{A}^{-}\right|^{2}=\frac{1}{8}|\phi|^{4}$, and the $C^{0}$ estimate $|\phi|^{2} \leqslant-s_{g}$ which follows from the equations and the Weitzenböck formula for the Dirac operator.

Combining the two inequalities, we conclude $\sigma(X) \geqslant 0$.

If $\sigma(X)=0$, then all the inequalities in the above calculation must be equalities. In particular, $F_{A}^{+}=0$ and $\phi$ is covariantly constant with $|\phi|^{2}=-s_{g}>0$. The Seiberg-Witten curvature equation then shows that $F_{A}$ is a parallel 2-form which, on $\bar{X}$, is $g$-self-dual, and is therefore a Kähler form compatible with $g$.

Recall that in dimension 4 the curvature tensor has 4 irreducible components: the scalar curvature, the self-dual and anti-self-dual Weyl tensor, and the traceless Ricci tensor. For a Kähler-Einstein metric, the scalar curvature is constant, the self-dual Weyl tensor is parallel, and the traceless Ricci tensor vanishes. In our situation, $g$ is Kähler-Einstein on both $X$ and $\bar{X}$, so that both parts of the Weyl tensor must be parallel. Thus the full curvature tensor of $g$ is parallel, and $g$ is locally Hermitian symmetric. As $X$ satisfies $c_{1}^{2}(X)=2 c_{2}(X)>0$, Hirzebruch proportionality implies that the universal cover of $X$ is the polydisk.

Here is the main result about complex surfaces admitting a complex structure compatible with the other orientation.

THEOREM 2. Let $X$ be a compact complex surface admitting a complex structure for $\bar{X}$. Then $X$ (and $\bar{X}$ ) satisfies one of the following:

(1) $X$ is geometrically ruled, or

(2) the Chern numbers $c_{1}^{2}$ and $c_{2}$ of $X$ vanish, or

(3) $X$ is uniformised by the polydisk.

In particular, the signature of $X$ vanishes.

Proof. In $[\mathbf{2}, \mathbf{6}]$ it was proved that under the assumptions of the theorem, if (1) or (2) do not hold, then $X$ is a surface of general type. We need to show that it is uniformised by the polydisk.

For a surface of general type, the topological Euler characteristic $c_{2}$ is positive, so $\bar{X}$ cannot satisfy (2). As surfaces of general type have non-zero Seiberg-Witten invariants $[11,3]$, Proposition 1 implies that $\bar{X}$ is not ruled. Thus $\bar{X}$ is also of general type, and we can apply Theorem 1 to both $X$ and $\bar{X}$. It follows that they have signature zero and are uniformised by the polydisk.

The surfaces occurring in Theorem 2 can be classified completely under suitable additional hypotheses about the fundamental group, about the existence of Kähler-Einstein metrics, or about the Kodaira dimension, as in the following three corollaries.

The first corollary proves a strong version of Conjecture 0.2 stated in [6]. 
COROLLARY 1. If two compact complex surfaces with finite fundamental groups are orientation-reversing diffeomorphic, then they are geometrically ruled. In particular, they are smooth 2-sphere bundles over the 2-sphere and are simply connected.

Proof. If $X$ and $\bar{X}$ are complex but not geometrically ruled, then either $\chi(X)=0$ or they are of general type. The first case is not possible if $b_{1}(X)=0$. In the second case the fundamental group is infinite by Theorem 2.

Another consequence of Theorem 2 is the classification of surfaces admitting Kähler-Einstein metrics for both orientations.

COROLlary 2. Suppose that $X$ and $\bar{X}$ each admit a Kähler-Einstein metric (not necessarily the same one). Then $X$ (and $\bar{X}$ ) is $S^{2} \times S^{2}$, or a compact quotient of the polydisk, or finitely covered by a complex torus. (All these cases occur, and the Kähler-Einstein metric is compatible with both orientations.)

Proof. As both $X$ and $\bar{X}$ are complex, we can apply Theorem 2. In case (1) we have either $S^{2} \times S^{2}$, or the blowup of the projective plane in one point. The latter admits no Kähler-Einstein metric because its automorphism group is not reductive. In case (2), the Cheeger-Gromoll splitting theorem for Ricci-flat manifolds implies that $X$ is finitely covered by a torus or a $K 3$ surface. The latter case is impossible, because the $K 3$ surface has positive Euler characteristic (and contains spheres of negative selfintersection). In case (3) there is nothing to prove.

Our final application of Theorem 2 is a uniformisation result characterising in terms of locally homogeneous Thurston geometries the surfaces with non-negative Kodaira dimension admitting a complex structure for $\bar{X}$.

COROllary 3. Let $X$ be a complex surface admitting a complex structure for $\bar{X}$. If the Kodaira dimension of one of the two surfaces (equivalently, both of them) is nonnegative, then $X$ and $\bar{X}$ are in fact orientation-preserving diffeomorphic. Furthermore, they carry a Thurston geometry compatible with the complex structure. The following surfaces and geometries can and do occur:

- surfaces of general type with geometry $\mathbb{H} \times \mathbb{H}$,

- properly elliptic surfaces with even $b_{1}$ and with geometry $\mathbb{C} \times \mathbb{H}$,

- properly elliptic surfaces with odd $b_{1}$ and with geometry $\mathrm{SL}_{2} \times \mathbb{R}$,

- tori and hyperelliptic surfaces with geometry $\mathbb{C}^{2}$,

- Kodaira surfaces with geometry $\mathrm{Nil}^{3} \times \mathbb{R}$.

Proof. We consider the cases in Theorem 2, referring to [1] for the Kodaira classification of compact complex surfaces. In case (3) the surfaces clearly carry a geometry of type $\mathbb{H} \times \mathbb{H}$, and case (1) is excluded by the assumption on the Kodaira dimension.

In case (2), under the additional assumption on the Kodaira dimension, we have only tori and elliptic surfaces without singular fibres (other than multiple ones with smooth reduction), because the presence of singular fibres makes $c_{2}>0$. Tori carry a geometry of type $\mathbb{C}^{2}$. The elliptic surfaces all have good orbifold bases, again by the assumption on the Kodaira dimension, so the results of Wall [10] show 
that they are as stated in the corollary. Clearly, all these cases occur and admit orientation-reversing selfdiffeomorphisms.

Remark 2. The assumption on the Kodaira dimension is necessary for several reasons. First, there are many ruled surfaces which are not geometric; see [10]. Some of them, like the odd Hirzebruch surfaces, are not even homotopy equivalent to geometric surfaces. However, they do have orientation-reversing selfdiffeomorphisms.

For class VII surfaces, the conditions in Theorem 2 imply that $b_{2}=0$. Of course, Hopf surfaces give examples, and a few of these carry a geometry of type $S^{3} \times \mathbb{R}$, but most do not. If we assume that the class VII surfaces under consideration have no curves, then they carry solvable Lie group geometries; compare $[\mathbf{1 0}, \mathbf{5}]$.

AcKNowledgements. I am grateful to N. C. Leung for sending me a copy of his preprint [9].

\section{References}

1. W. Barth, C. Peters and A. VAn De Ven, Compact complex surfaces (Springer, Berlin, 1984).

2. A. Beauville, 'Surfaces complexes et orientation', Astérisque 126 (1985) 41-43.

3. S. K. Donaldson, 'The Seiberg-Witten equations and 4-manifold topology', Bull. Amer. Math. Soc. 33 (1996) 45-70.

4. R. FintuShel and R. J. Stern, 'Immersed spheres in 4-manifolds and the immersed Thom conjecture', Proc. Gökova Geometry-Topology Conference 1994, Turkish J. Math. 19 (2) (1995) 27-39.

5. D. KотSCHICK, 'Remarks on geometric structures on compact complex surfaces', Topology 31 (1992) 317-321.

6. D. KoтsCHick, 'Orientation-reversing homeomorphisms in surface geography', Math. Ann. 292 (1992) 375-381.

7. D. Kotschick and G. Matić, 'Embedded surfaces in four-manifolds, branched covers, and $S O(3)$ invariants', Math. Proc. Cambridge Philos. Soc. 117 (1995) 275-286.

8. D. Kotschick, J. W. Morgan and C. H. Taubes, 'Four-manifolds without symplectic structures but with non-trivial Seiberg-Witten invariants', Math. Res. Lett. 2 (1995) 119-124.

9. N. C. LeUnG, 'Seiberg Witten invariants and uniformization', Math. Ann., to appear.

10. C. T. C. WALL, 'Geometric structures on compact complex surfaces', Topology 25 (1986) 119-153.

11. E. Witten, 'Monopoles and four-manifolds', Math. Res. Lett. 1 (1994) 769-796.

Mathematisches Institut

Universität Basel

Rheinsprung 21

4051 Basel

Switzerland 